\title{
Original
}

\section{Driving Mobility, Older Adults, and Quality of Life}

\author{
Cynthia Owsley PhD \\ Department of Ophthalmology, School of Medicine, \\ University of Alabama at Birmingham, 700 S. 18th Street, Suite 609, \\ Birmingham,AL 35294-0009, USA \\ e-mail: owsley@eyes.uab.edu
}

\begin{abstract}
C. Owsley, Driving Mobility, Older Adults, and Quality of Life, Gerontechnology 2002; 1(4): 220 - 230. Driving mobility is a fundamental instrumental activity of daily living for many older adults throughout the world. There are proportionately more older drivers on the road than ever before, they have higher annual mileage than earlier older cohorts, and they have a higher crash and injury rate per mile driven than most other age groups. Driving cessation in older adults is associated with increased depression and social isolation. Visual, cognitive, and physical impairments and the medical conditions that engender them elevate crash risk and impair on-road performance in older drivers. Older adults report more difficulty on the road than younger adults and these selfrecognized problems are associated with their functional impairments. These recent research findings underscore the pressing need for public health and technological research focused on enhancing the safety of older drivers while also preserving their mobility and quality of life.
\end{abstract}

Keywords: driving, mobility, functional impairment, aging, driver safety

Mobility can be defined as a person's purposeful movement through the environment from one place to another. It typically involves moving through space to accomplish some task or achieve some goal that cannot be reached where one already resides. It is a fundamental instrumental activity of daily living. Mobility is linked to a person's physical and psychological well-being. Limitation in mobility is associated with decreased quality of life, basic physiological functions, independent living, and personal autonomy, and increases the need for both formal and informal care ${ }^{1-2}$. Thus, mobility impairment, and the diseases and health conditions that engender it, can have serious ramifications for the affected individual as well as his/her family support system. Even further, mobility impairment is a serious challenge for the society in which the affected individuals live, since those who have impaired mobility, like everyone else in the society, have daily needs to move around in their immediate and more distant environment.

It is within this broad context that driving by older adults must be addressed, since driving is a typical type of mobility in many societies. The elderly represent the most rapidly growing segment of the driving population in many countries ${ }^{3}$. It has been estimated that in the U.S. by the year 2024, one of four drivers will be over the age of 65 . Older drivers today are also driving more miles per year than previous cohorts of older adults. For every 100,000 miles driven, the crash rate of 
older adults is twice that of younger drivers ${ }^{4}$. Once they are involved in a crash, older drivers are more likely than younger adults to incur injuries that lead to disability or death ${ }^{5-7}$. Motor vehicle collision is the second most common reason for older adults' visits to emergency departments for injuries ${ }^{8}$. In short then, there are proportionately more older drivers on the road than ever before, and they have a higher crash and injury rate than most other age groups.

Possession of a driver's license in many societies is itself meaningful, implying personal independence and self-control over one's life. Studies have shown that older drivers tend to continue driving as long as it is possible for them to do so, and although they may cut down on their frequency of travel and mileage behind the wheel, they resist any change in their preferred mode of travel $^{9}$. Driving cessation in older adults, as studied in prospective studies, has been associated with an increase in depressive symptoms and social isolation, and thus is related to overall quality of life for many older persons.

This gives rise to the realization that there are pressing public health and technological needs with respect to enhancing the safety while preserving the mobility of older drivers. In recent years there has been a great deal of progress in identifying the types of functional impairments that elevate crash risk in older drivers. Safe driving relies on a number of key skills that involve visual, cognitive $e^{10,11}$, and physical capabilities. Impairments in these functional capabilities can occur at any age, but are more prevalent in the older adult population. A growing body of research over the past decade has demonstrated that certain types of functional impairment in older drivers elevate their crash risk and have a negative impact on driving performance (summarized below). A clear understanding about how diminished capabilities contribute to crash risk in older drivers will greatly facilitate society's efforts to develop strategies to reduce crash rate in this population and to enhance driving mobility.

\section{VISUAL IMPAIRMENT}

The literature addressing the relationship between visual impairment and driving is substantial, with one recent review listing about 200 published articles on the topic, many from the past decade ${ }^{12}$. Probably the most common type of study involves examining associations between visual acuity and driving performance, since acuity is the most ubiquitous visual screening test used by state licensing agencies for the determination of driving fitness. Most studies have reported positive, weak associations between visual acuity and crash involvement or no associations at all ${ }^{13-25}$. Thus there is little support from the literature that a strong association exists between visual acuity and unsafe driving in the older adult driving population. However, visual acuity is related to the performance of certain driving tasks. For example, simulated acuity impairment (from induced optical blur) is related to decrements in road sign recognition and road hazard avoidance $^{26}$.

\section{Visual Acuity}

Given that visual acuity is the most common vision screening test for licensure, it seems paradoxical at first glance that research generally does not support the conclusion that acuity tests can effectively identify high risk drivers. There are several possible reasons why studies have generally failed to find strong associations between visual acuity and crash risk, as discussed in detail elsewhe$\mathrm{re}^{12,27-28}$. A prominent reason undoubtedly stems from the components and task demands of the driving task itself. Driving along a roadway and through intersections involves the simultaneous use of central and peripheral vision and requires monitoring of multiple sources of visual information, all in the midst of a visually cluttered array whose elements are in motion. Because of the visually complex nature of driving, other types of visual processing impairments even in the 
presence of good acuity could jeopardize safe driving, as discussed below. An acuity screener would fail to identify individuals with visual processing impairments as high risk.

\section{Peripheral Vision}

Several studies have examined the role of deficits in peripheral vision in driver safety and performance. One of the most quoted studies is a California study ${ }^{29}$ finding that crash and violation rates were twice as high among those with severe binocular field loss compared to those without any loss, adjusting for annual mileage. More recently a study reported a similar finding in that those older drivers with impaired peripheral vision in the better functioning eye were two times more likely to be crash involved compared to those with good peripheral vision in the better eye ${ }^{14}$. However, a number of other studies $^{13,}$ 20, 30-31 have not reported elevated crash rates for those with visual field impairments. An important consideration in comparing these results is that the definition of impairment differs across the studies. The California study ${ }^{29}$ defined impairment as severe binocular field loss, whereas most other studies defined it in a less impaired fashion. Visual field impairment also impacts certain aspects of driving performance. Visual field impairment compromises some (e.g., identification of road signs, avoid obstacles, reaction time) but not all (e.g., speed estimation, stopping distance) aspects of driving performance ${ }^{32-34}$.

\section{Contrast Sensitivity}

A primary function of the visual system is to process contrast information, which underlies the ability to see patterns in the environment. There are comparatively fewer studies on the role of contrast sensitivity in driving, as compared to the literature on acuity and visual fields. A few studies have reported associations between contrast sensitivity and crash involvement. Greater impairment in contrast sensitivity has been linked to a higher number of at-fault crashes in the prior five years ${ }^{14}$, and in the subsequent three years $^{35}$, although these associations were not adjusted for confounding factors. Wood and colleagues have examined the impact of simulated contrast sensitivity on driving performance on a closed road course ${ }^{32-33}$. Better contrast sensitivity was associated with better driving skills. Along these lines, Rubin and colleagues $^{36}$ reported that older drivers reporting difficulties with day and evening driving had worse contrast sensitivity. Contrast sensitivity is linked to road sign recognition ${ }^{37-39}$. A common cause of contrast sensitivity loss in old adults is cataract, a prevalent eye condition in the elderly that involves an opacification of the crystaline lens. Almost half of adults exhibit early cataract by age 75, and approximately one-quarter with more advanced cataract ${ }^{40-41}$. A recent study ${ }^{42}$ evaluated the role of contrast sensitivity impairment in elevating crash risk in a large sample of older adults with clinically significant cataract (20/40 or worse in at least one eye). Those older drivers with a history of recent crash involvement were eight times more likely to have severe contrast sensitivity impairment as compared to those older drivers who were crash-free. Impaired acuity was unrelated to crash involvement in cataractous drivers when contrast sensitivity deficits were adjusted for. Given the importance of image contrast in pattern vision and evidence that contrast sensitivity deficits underlie driving problems in older drivers with cataract, this is an area deserving of further study.

\section{Color Vision}

There are a number of other aspects of visual function that have been considered with respect to driving. It is well established that older adults typically experience impairments in color discrimination ${ }^{43}$, primarily along the tritan or 'blue-yellow' axis, so the question of how this impacts their driving is relevant. The underlying rationale for color vision testing in both personal and commercial driver licensing is not the belief that color deficiency is a major risk factor for crash involvement; 
rather, color vision screening is simply a way to insure that drivers can obey color traffic control devices. The critical cues on the road usually can be obtained through multiple sources of information (e.g., luminance, position, pattern), so drivers with color vision anomalies do not experience serious difficulty in traffic signal recognition. Vingrys and Cole's comprehensive review of this literatu$\mathrm{re}^{44}$ indicates that the vast majority of studies on color vision and road safety support this conclusion, finding no association between color deficiencies and vehicle crash involvement or impaired driving performance. Owing to this overwhelming wealth of evidence, it is reasonable to conclude that color deficiency by itself does not elevate crash risk in personal or commercial drivers, or for older drivers with modest color discrimination problems.

\section{Disability Glare}

Disability glare problems are discussed as a serious threat to the safety of older road users $^{45}$, but one is hard-pressed to identify actual studies that scientifically confirm this notion. This failure to find an association between glare and road safety may be due to methodological difficulties in defining 'glare' and in measuring a multi-faceted phenomenon, as well as to a poor understanding of what people mean when they say they have 'glare' problems.

\section{Motion Perception}

The visual world of the driver is in motion, and in this sense, stationary test targets in driving assessment tests are not very representative of the visual scene. Studies from the 1960s and 1970s, which included both static and dynamic acuity measurements, have generally found that dynamic targets had stronger univariate associations with crash involvement than did static targets ${ }^{13,46}$. However the associations between dynamic visual acuity and driver safety were still weak. It has been noted that dynamic acuity deteriorates more rapidly with age. Furthermore, individuals with the same static acuity can have widely divergent dynamic acuity. At the very least, tests of sensitivity for dynamic visual events require a closer look in terms of their association to driver safety and performance.

The role of eye movement disorders in driving is largely an untouched research area. Prior research on normally-sighted drivers indicates that experienced drivers continuously scan the road scene for useful information $^{47}$. Older drivers with restrictions in the ability to turn their heads experience a restriction in the distances at which approaching traffic could be brought into the central visual field for visual inspection ${ }^{48}$. Motion perception and optical flow phenomena such as 'heading' have a great deal of face validity to the driving task, but little work has addressed how impairments in motion processing may affect driving performance and safety. Three decades ago a study ${ }^{45}$ showed that performance in a motion perception task was one of the best correlates of self-reported crash involvement among a large battery of vision tests, but the relationship was still weak. This study also found that acuity under low illumination was related to nighttime crash involvement, but again the link was weak. It has been suggested that drivers' errors and crashes at night may stem from their lack of awareness of perceptual limitations that occur in low light ${ }^{49}$. However, as will discussed later, older drivers appear to be quite good at self-regulation, and one of the earliest modifications to driving habits is the elimination of night driving.

\section{Eye Disease}

Most vision-threatening eye conditions that occur in the elderly are bilateral in nature, i.e. affecting both eyes (e.g., cataract, age-related macular degeneration, glaucoma, diabetic retinopathy). However, the severity and rate of progression of the condition can vary greatly between the two eyes, allowing for situations where one eye retains good function while the other eye is seriously impaired. 
This issue may be highly relevant in understanding crash risk in older drivers given the results of a recent study on older drivers with cataract $^{42}$. This study showed that vision impairment need only be present in one eye to substantially elevate crash risk. A handful of studies have specifically examined the safety and driving performance capabilities of 'monocular' drivers. In these studies, monocularity has been defined in a variety of ways. Although in these studies one eye has good acuity or good peripheral vision (often not precisely defined), the fellow eye can vary across studies from no vision at all, to acuity worse than $20 / 200$ or significant scotomas in the visual field, to no definition of monocularity given whatsoever. Thus, interpreting study results and summarizing across studies can be difficult. With respect to actual driving performance, simulated monocular vision, by occluding one eye, did not impact driving maneuvers on a closed-road course ${ }^{32}$ ${ }^{33}$. With respect to safety, those drivers with monocular field loss did not have an elevated crash rate compared to a control group of drivers with normal visual fields in both eyes $^{29}$. Not all studies are consistent with these findings however ${ }^{50-51}$, finding higher rates of violations and mishaps among monocular drivers as compared to those with normal vision in both eyes. A growing body of evidence suggests that monocularity, particularly visual acuity impairment worse than $20 / 200$ in one eye, elevates crash risk among commercial drivers who are exposed to high levels of driving often in intense traffic situations ${ }^{52-54}$. A study performed by the California Department of Motor Vehicles ${ }^{54}$ on the safety of heavy-vehicle truck drivers found that those who were monocular (acuity worse than 20/200 in one eye) had more total convictions and crashes than did non-visuallyimpaired drivers. Yet this topic remains controversial. For example, a recent study ${ }^{55}$ that assessed driving performance in both monocular and binocular commercial drivers reported there were no group differences in the safety with which most day-to-day driving driving maneuvers were performed.

\section{VISUO-COGNITIVE AND COGNITIVE IMPAIRMENT}

Because driving is a complex visual/cognitive task, it is unlikely that an assessment of visual sensory impairment and the diagnosis of eye disease would alone be sufficient to identify those at elevated risk for crash involvement. Visual information processing skills, not only visual sensory thresholds, have a great deal of face validity to the execution of safe driving practices. One such skill that appears to be relevant is visual attention. It is interesting that several studies from the early 1970s implied that impaired visual attentional abilities were linked to crash involvement ${ }^{56-58}$, but this finding was not further explored until more recently.

During the late 1980's, a task called the Useful Field of View was developed which assesses the visual field area over which one can use rapidly presented information ${ }^{59-62}$. Unlike conventional measures of visual field, which assess visual sensory sensitivity, the Useful Field of View test additionally relies on higher-order processing skills such as selective and divided attention and rapid visual processing speed. Over the past decade, in excess of 50 published articles have evaluated the UFOV ${ }^{\circledast}$ test in the context of driving competence $^{63}$. For example, reduction in the useful field of view in older drivers is associated with a history of at-fault crash involvement $^{14,25}$ and injurious crash involvement ${ }^{64}$. Those drivers with the most severe restrictions tended to have the highest number of crashes during the prior five years ${ }^{14}$. In a prospective study 30 older drivers with a $40 \%$ or greater impairment in the useful field of view were two times more likely to incur a crash during the three years of follow-up, after adjusting for age, sex, race, chronic medical conditions, mental status, and driving exposure. This association was primarily mediated by difficulty in dividing attention under brief target durations. It is noteworthy that in this study useful field of view impairment was the only type of visual deficit that was related to future crash involvement; 
deficits in acuity, contrast sensitivity, and visual field sensitivity were unrelated to future crashes.

The UFOV ${ }^{\circledR}$ test has also been used to study crash proneness in the Alzheimer's disease population. Studies indicate that in drivers with $A D$, that useful field of view reduction is one of the best predictors of crash-involvement in a simulator and poor on-road performance in a driving test, when compared to other cognitive tests ${ }^{65-67}$. These studies imply that visual attention and visual processing speed are critical considerations when evaluating safe driving skills and may be better screening tests for detecting high-risk older drivers than visual sensory tests, a practical issue worthy of focused investigation.

Impaired performance on other tests of higher order visual processing abilities have also been related to crash involvement and impaired driving performance, underscoring the importance of assessing visual skills beyond basic capabilities. Studies have reported associations between unsafe driving and deficits in visual search and sequencing abilities $^{65}$, selective attention tasks ${ }^{19,65}$, spatial memory $^{67-68}$ the perception of three-dimensional structure from motion ${ }^{68}$, and the Trails test ${ }^{14,66,69-71}$. The strength of the association between driving competence measures and the visuo-cognitive measures is consistently much stronger than with visual sensory function alone.

\section{PHYSICAL IMPAIRMENT}

Controlling a vehicle in a safe fashion obviously depends on the successful execution and continuous monitoring of physical behaviors. Several aspects of motor ability that on face validity appear to be important for the driving task are strength, coordination (both gross and fine), range of motion of head/neck, arms, and legs including the overall flexibility of the extremities, and balance and gait (e.g., in getting in and out of a vehicle, sitting in a stable fashion behind the wheel). As summarized by Marottoli ${ }^{72}$, what is most striking about the area of physical function and driving in older adults is that relatively little is known about how physical function deficits in the elderly impact their driving safety and driving behavior. There is little to no information available about what minimum levels of physical functioning are necessary for safe driving. Reductions in strength could theoretically be a problem when turning the steering wheel or shifting the gear lever, however, most new vehicles' controls are designed now to accommodate a wide range of driver strength to facilitate ease of operation. Retchin and colleagues ${ }^{73}$ found a bivariate association between grip strength and driving frequency among older veterans, suggesting that some older adults with impaired strength may avoid driving. Range of motion with respect to the head and neck is especially important for checking for vehicles, pedestrians, and other obstacles in the roadway environment. It has been shown that older drivers with restricted ability to turn their heads (e.g., from arthritis) are limited in the distances at which approaching traffic can be brought into the central visual field for visual inspection, and that limited neck rotation is a risk factor for future crash involvement $^{19}$. Gait and balance problems may be especially a problem when getting in and out of vehicles, which may hamper the use of one's vehicle, even though the person may be safe behind the wheel, an issue worthy of study. In terms of lower extremity problems, foot abnormalities in older adults including increased foot reaction time have been associated with the occurrence of crashes and moving violations in older drivers ${ }^{23,74}$, implying that these problems may hamper the ability to maneuver between the accelerator and the brake in a timely fashion. Capabilities associated with physical activity have also been related to older adults' driving safety. Crash- or violation-involved older drivers walked fewer blocks on the average day ${ }^{23}$ and reported difficulty with physical activities such as walking a mile, opening a jar and doing yard work or light 
housework and were more likely to have a history of falls $\mathrm{s}^{75-76}$, compared to those who were not crash- or violation-involved. This implies that physical difficulties with other instrumental activities of daily living may serve as markers or warning signs that person may also be having problems with driving. It has long been known that reaction time to a visual stimulus is increased on average in older adults compared to younger adults when measured in a laboratory setting, however, slow reaction time has not been consistently identified as a risk factor for older adults' crash involvement. This may be because of compensatory behaviors like driving more slowly and avoiding heavy traffic areas, although these potential coping strategies deserve further investigation in terms of their role in minimizing crash risk among the elderly.

\section{DRIVING DIFFICULTIES AND SELF- REGULATION BY OLDER DRIVERS}

There is evidence that the majority of older drivers modify or self-regulate their driving habits in certain driving situations. For example, older drivers with declining visual and/or cognitive function limit their driving exposure in driving situations which are generally believed to be more difficult (e.g., rain, night, heavy traffic, rush hour $)^{77-78}$. There are several potential reasons why older adults modify their driving habits in later life, such as they may have more flexible daily schedules and thus have greater freedom to choose when and where they drive or they may have less need to drive on a daily basis. There is growing evidence that some older adults may limit their driving because they recognize that they have functional impairments that may prompt safety concerns. For example, older drivers with clinical significant cataract (impairing visual acuity to $20 / 40$ or worse) are more likely to report driving difficulty and limit their driving exposure than those older drivers who do not have cataract 79-80. Older drivers with impairments in contrast sensitivity, increased disability glare, slowed visual processing speed, and/or divi- ded attention problems report more avoidance of challenging driving situations than those without these problems ${ }^{77,80}$.

Although it appears that many older adults have self-awareness about the implications of their visual impairments for safe driving, it also appears that there are substantial numbers of older drivers with impairments who do not understand the link. A recent study found that in a large sample of visually impaired drivers who still met the legal requirements for driving, over half believed that their vision problems were not likely to put them at increased risk for crashing ${ }^{81}$. Threequarters of these drivers reported never or rarely avoiding challenging driving situations. However, what was particularly interesting is that eighty percent acknowledged that they would feel more protected from crashing if they avoided certain situations, and also felt that they had high self-efficacy in their ability to self-regulate. These findings imply that functionally impaired older drivers who do not currently engage in 'self-protective' driving behaviors may have the capacity to change their driving strategies and engage in self-regulation on the road. A recent study evaluating the impact of an educational intervention on high-risk visually-impaired drivers implied that they benefited from the educational program in that following their participation in the program, they increased their avoidance of challenging driving situations and reduced their driving exposure ${ }^{82-83}$. The primary outcomes in this study were based on self-report measures and thus a critical future step is to examine whether the education intervention has an impact on the safety of high-risk older drivers by reducing their rate of crash involvement, an issue currently under investigation.

\section{SUMMARY AND CONCLUSIONS}

Driving is the preferred mode of transportation for the majority of older adults in many countries. As such, many older adults depend on the automobile to meet their 


\section{Driving Mobility}

mobility needs. This is an important issue, since sustaining mobility is linked to physical and psychological well being and a satisfying quality of life. Nevertheless, as the fastest growing age group of drivers, their higher rate of crash involvement per mile driven compared to younger drivers needs to be addressed in the interest of both public health and public safety. The recent years have brought us many studies describing the visual, cognitive, and physical risk factors for crash involvement and impaired driving performance in older drivers. In addition, we know that some older drivers will self-regulate their driving to safer circumstances, either on their own or if they are educated about the potential protective benefit of these behaviors. It is time now for research to focus on developing and evaluating interventions to enhance driving safety while preserving driving mobility and quality of life whenever possible. As we will see in the other articles in this volume, technological developments and solutions have great promise for eventually meeting this goal.

\section{ACKNOWLEDGEMENT}

This research program is funded by National Institute on Aging/NIH grant P50-AG11684 (Edward R. Roybal Center for Research in Applied Gerontology), the Centers for Disease Control (contract \#166804), Research to Prevent Blindness, Inc., and General Motors Corporation pursuant to an agreement between General Motors and the United States Department of Transportation.

\section{References}

1. Ettinger WHJ. Immobility. In: Hazzard WR, Bieman EL, Blass JP, Ettinger WHJ, Halter JB, editors, Principles of Geriatric Medicine and Gerontology, Third Edition. New York: McGraw-Hill; 1994; pp1307-1311

2. Tinetti ME, Inouye SK, Gill TM, Doucette JT. Shared risk factors for falls, incontinence, and functional dependence: Unifying the approach to geriatric syndromes. JAMA 1995;273(17):1348-1353

3. National Highway Traffic Safety Administration. Conference on Research and Development Needed to Improve Safety and Mobility of Older Drivers. Washington, D.C.:
U.S. Department of Transportation; 1989.

DOT HS 807316

4. Transportation Research Board.

Transportation in an Aging Society. Vol 1.

Washington D.C.: National Research Council; 1988

5. McCoy GF, Johnson RA, Duthie RB. Injury to the elderly in road traffic accidents. Journal of Trauma 1989;29:494-497

6. Barancik JI, Chatterjee BF, Greene-Cadden YC, Michenzi EM. Motor vehicle trauma in northeastern Ohio. I. Incidence and outcome by age, sex, road-use category. American Journal of Epidemiology 1986;74:473-478

7. Evans L. Risk of fatality from physical trauma versus sex and age. The Journal of Trauma 1988;28(3):368-378

8. Fingerhut LA, Warner M. Injury Chartbook. Health, United States, 1996-97. Hyattsville, Maryland: National Center for Health Statistics; 1997

9. Jette $A M$, Branch LG. A ten-year follow-up of driving patterns among the communitydwelling elderly. Human Factors 1992;34:2531

10. Marottoli RA, de Leon CFM, Glass TA, Leon CFM de, Glass TA, Williams CS, Cooney LM, Berkman F, Tinetti ME. Driving cessation and increased depressive symptoms: Prospective evidence from the New Haven EPESE. Journal of the American Geriatrics Society 1997;45:202-206

11. Fonda SJ, Wallace RB, Herzog AR. Changes in driving patterns and worsening depressive symptoms among older adults. Journal of Gerontology: Social Sciences 2001;56B(6):S343-S351

12. Owsley C, McGwin GJ. Vision impairment and driving. Survey of Ophthalmology 1999;43(6):535-550

13. Hills BL, Burg A. A reanalysis of California driver vision data:

General findings: Crowthorn, England: Transport and Road Research Laboratory; 1977; p 768

14. Ball K, Owsley C, Sloane ME, Roenker DL, Bruni JR. Visual attention problems as a predictor of vehicle crashes in older drivers. Investigative Ophthalmology and Visual Science 1993;34(11):3110-3123

15. Davison PA. Inter-relationships between British drivers' visual abilities, age and road accident histories. Ophthalmic and Physiological Optics 1985;5(2):195-204

16. Henderson R, Burg A. Vision and audition in driving. Washington DC: U.S. Department of Transportation; 1974

17. Hofstetter HW. Visual acuity and highway accidents. Journal of the American 
Optometric Association 1976;47(7):887-893

18. Humphriss D. Three South African studies on the relation between road accidents and drivers' vision. Ophthalmic and Physiological Optics 1987;7(1):73-79

19. Marottoli RA, Richardson ED, Stowe $M H$, Miller EG, Brass LM, Cooney LM, Tinetti ME. Development of a test battery to identify older drivers at risk for self-reported adverse driving events. Journal of the American Geriatrics Society 1998;46:562-568

20. Decina LE, Staplin L. Retrospective evaluation of alternative vision screening criteria for older and younger drivers. Accident Analysis and Prevention 1993;25(3):267-275

21. Gresset JA, Meyer FM. Risk of accidents among elderly car drivers with visual acuity equal to $6 / 12$ or $6 / 15$ and lack of binocular vision. Ophthalmic and Physiological Optics 1994;14:33-37

22. Johansson $K$, Bronge $L$, Lundberg $C$, Persson A, Seideman M, Viitanen M. Can a physician recognize an older driver with increased crash risk potential? Journal of the American Geriatric Society 1996;44:1198-1204

23. Marottoli RA, Cooney Jr. LM, Wagner DR, Doucette J, Tinetti ME. Predictors of automobile crashes and moving violations among elderly drivers. Annals of Internal Medicine 1994;121(11):842-846

24. McCloskey LW, Koepsell TD, Wolf ME, Buchner DM. Motor vehicle collision injuries and sensory impairments of older drivers. Age and Ageing 1994;23:267-273

25. Owsley C, Ball K, Sloane ME, Roenker DL, Bruni JR. Visual/cognitive correlates of vehicle accidents in older drivers. Psychology and Aging 1991;6(3):403-415

26. Higgins KE, Wood J, Tait A. Vision and driving: Selective effect of optical blur on different driving tasks. Human Factors 1998;41(2):224-232

27. Shinar D, Schieber F. Visual requirements for safety and mobility of older drivers. Human Factors 1991;33:507-519

28. Ball K, Owsley C. Identifying correlates of accident involvement for the older driver. Human Factors 1991;33:583-595

29. Johnson CA, Keltner JL. Incidence of visual field loss in 20,000 eyes and its relationship to driving performance. Archives of Ophthalmology 1983;101:371-375

30. Owsley C, Ball K, McGwin G, Sloane ME, Roenker DL, White MF, Overley ET. Visual processing impairment and risk of motor vehicle crash among older adults. JAMA 1998;279:1083-1088

31. Burg A. Lateral visual field as related to age and sex. Journal of Applied Psychology 1968:52:10-15

32. Wood JM, Troutbeck R. Effect of restriction of the binocular visual field on driving performance. Ophthalmic and Physiological Optics 1992;12:291-298

33. Wood JM, Dique T, Troutbeck R. The effect of artificial visual impairment on functional visual fields and driving performance. Clinical Vision Science 1993;8(6):563-575

34. Wood JM, Troutbeck R. Elderly drivers and simulated visual impairment. Optometry and Vision Science. 1995;72(2):115-124

35. Owsley C. Vision and driving in the elderly. Optometry and Vision Science 1994;71(4):727-735

36. Rubin GS, Roche KB, Prasada-Rao P, Fried LP. Visual impairment and disability in older adults. Optometry and Vision Science 1994;71(12):750-760

37. Evans DW, Ginsburg AP. Contrast sensitivity predicts age-related differences in highway sign discriminability. Human Factors 1985;27:637-642

38. Babbitt Kline TJ, Ghali LM, Kline DW, Brown S. Visibility distance of highway signs among young, middle-aged, and older observers: icons are better than text. Human Factors 1990;32(5):609-619

39. Owsley C, Sloane ME. Contrast sensitivity and the perception of 'real-world' targets. British Journal of Ophthalmology 1987;71:791-796

40. Kahn HA, Leibowitz HM, Ganley JP, Kini $M M$, Colton T, Nickerson RS, Dawber TR. The Framingham eye study, I. Outline and major prevalence findings. American Journal of Epidemiology 1977;106(1):17-41

41. Klein BEK, Klein R, Linton KLP. Prevalence of age-related lens opacities in a population: The Beaver Dam Eye Study. Ophthalomology 1992;99:546-552

42. Owsley C, Stalvey BT, Wells J, Sloane ME, McGwin J, G. Visual risk factors for crash involvement in older drivers with cataract. Archives of Ophthalmology 2001;119:881887

43. Knoblauch K, Saunders F, Kusuda M, Hynes $R$, Podgor $M$, Higgins KE, deMonastereo FM. Age and illuminance effects in the Farnsworth-Munsell 100-hue test. Applied Optics 1987;26:1441-1448

44. Vingrys AJ, Cole BL. Are colour vision standards justified for the transport industry? Ophthalmic and Physiological Optics 1988;8:257-274

45. Wolbarsht ML. Tests for glare sensitivity and peripheral vision in driver applicants. Journal 


\section{Driving Mobility}

of Safety Research 1977;9:128-139

46. Shinar D. Driver visual limitations: Diagnosis and treatment. Washington DC: U.S. Department of Transportation; 1977. DOT HS 51275

47. Mourant RR, Rockwell TH. Strategies of visual search by novice and experienced drivers. Human Factors 1972; 14:325-335

48. Isler RB, Parsonson BS, Hansson GJ. Age related effects of restricted head movements on the useful field of view of drivers. Accident Analysis and Prevention 1997;29(6):793-801

49. Leibowitz HW, Owens DA. Nighttime driving accidents and selective degradation. Science 1977;197:422-423

50. Liesmaa $M$. The influence of driver's vision in relation to his driving ability. Optician 1973;166:10-13

51. Keeney $\mathrm{AH}$. Relationship of ocular pathology and driving impairment. Transactions of the American Academy of Ophthalmology and Otolaryngology 1968;72:737-740

52. Maag U, Vanasse C, Dionne G, LabergeNadeua C. Taxi-drivers' accidents: How binocular vision problems are related to their rate and severity in terms of the number of victims. Accident Analysis and Preventioin 1997;29:217-224

53. Laberge-Nadeau C, Dionne G, Maag U, Desjardins D, Vanasse C, Ekoe J-M. Medical conditions and the severity of commercial motor vehicle drivers' road accidents.

Accident Analysis and Prevention 1996;28:43-51

54. Roger PN, Ratz M, Janke MK. Accident and conviction rates of visually impaired heavyvehicle operators: State of California Department of Motor Venhicles, Research and Development Office, Sacremento, CA; 1987

55. McKnight AJ, Shinar D, Hilburn B. The visual and driving performance of monocular and binocular heavy-duty truck drivers. Accident analysis and Prevention 1991;23(4):225-237

56. Barrett GV, Mihal WL, Panek PE, Sterns HL, Alexander RA. Information-processing skills predictive of accident involvement for younger and older commercial drivers. Industrial Gerontology 1977;4:173-182

57. Kahneman D, Ben-Ishai R, Lotan M. Relation of a test of attention to road accidents. Journal of Applied Psychology 1973;58:113-115

58. Mihal WL, Barrett GV. Individual differences in perceptual information

processing and their relation to automobile accident involvement. Journal of Applied Psychology 1976;61(2):229-233

59. Ball KK, Roenker DL, Bruni JR.
Developmental changes in attention and visual search throughout adulthood. In: Enns JT, editor, The Development of Attention: Research and Theory. Amsterdam: Elsevier Science; 1990; pp 489-507

60. Ball K, Beard BL, Roenker DL, Miller RL, Griggs DS. Age and visual search: expanding the useful field of view. Journal of the Optical Society of America A 1988;5:22102219

61. Sekuler R, Ball K. Visual localization: age and practice. Journal of the Optical Society of America A 1986;3(6):864-867

62. Sanders AF. Some aspects of the selective process in the functional field of view. Ergonomics 1970;13:101-117

63. Ball K, Wadley V, Edwards J, Goode K, Myers $R$, Frankel $M$. Useful field of view and driving competence. Human Factors, in press.

64. Owsley C, McGwin G, Jr., Ball K. Vision impairment, eye disease, and injurious motor vehicle crashes in the elderly. Ophthalmic Epidemiology 1998;5:101-113

65. Duchek JM, Hunt L, Ball K, Buckles V, Morris $J C$. Attention and driving performance in Alzheimer's Disease. Journal of Gerontology: Psychological Sciences 1998;53B(2):130-141

66. Cushman LA. Cognitive capacity and concurrent driving performance in older drivers. IATSS Research 1996;20(1):38-45

67. Rizzo M, Reinach S, McGehee D, Dawson J. Simulated car crashes and crash predictors in drivers with Alzheimer disease. Archives of Neurology 1997;54:545-551

68. Odenheimer $G L$, Beaudet $M$, Jette $A M$, Albert MS, Grande L, Minaker KL. Performance-based driving evaluation of the elderly driver: Safety, reliability, and validity. Journal of Gerontology: Medical Sciences. 1994;49(4):M153-M159

69. Stutts JC. Do older drivers with visual and cognitive impairments drive less? Journal of the American Geriatrics Society. 1998;46:854-861.

70. Tarawneh MS, McCoy PT, Bishu RR, Ballard $J$. Factors associated with driving performance of older drivers. Transportation Research Record 1993;1405:64-71

71. Cooper PJ, Tallman K, Tuokko H, Beattie BL. Vehicle crash involvement and cognitive deficit in older drivers. Journal of Safety Research 1993;24:9-17

72. Marottoli RA, Drickamer MA. Psychomotor mobility and the elderly driver. In: Retchin SM, ed. Clinics in Geriatric Medicine: Medical Considerations in the Older Driver. Philadelphia: W.B. Saunders; 1993; pp 403411 
73. Retchin SM, Cox J, Fox M, Irwin L. Performance-based measurements among elderly drivers and nondrivers. Journal of American Geriatrics Society 1988;36:813-819

74. Margolis KL, Kerani RP, McGovern $P$, Songer T, Cauley JA, Ensrud KE for the Study of Osteoporotic Fractures Research Group. Risk factors for motor vehicle crashes in older women. Journal of Gerontology: Medical Sciences 2002;57A:M186-M191

75. Sims RV, Owsley C, Allman RM, Ball K, Smoot TM. A preliminary assessment of the medical and functional factors associated with vehicle crashes in older adults. Journal of the American Geriatrics Society 1998;46:556-561

76. Sims RV, McGwin G, Jr., Allman RM, Ball K, Owsley C. Exploratory study of incident vehicle crashes among older drivers. Journal of Gerontology: Medical Sciences 2000;55A(1):M22-M27

77. Ball K, Owsley C, Stalvey B, Roenker DL, Graves $M$. Driving avoidance and functional impairment in older drivers. Accident Analysis and Prevention 1998;30:313-322
78. Schlag B. Elderly drivers in Germany - Fitness and driving behavior. Accident Analysis and Prevention 1993;25(1):47-55

79. Owsley C, Stalvey B, Wells J, Sloane ME. Older drivers and cataract: Driving habits and crash risk. Journal of Gerontology: Medical Sciences 1999;54A(4):M203-M211

80. McGwin GJ, Chapman V, Owsley C. Visual risk factors for driving difficulty in older drivers. Accident Analysis and Prevention 2000;32:735-744

81. Stalvey BT, Owsley C. Self-perceptions and current practices of high-risk older drivers: Implications for driver safety interventions. Journal of Health Psychology 2000;5(4):441456

82. Owsley C, Stalvey BT, Phillips JM. The efficacy of an educational intervention in promoting self-regulation among high-risk older drivers. Accident Analysis and Prevention. In press

83. Stalvey BT, Owsley C. The development of a theory-based educational curriculum to promote self-regulation among high-risk older drivers. Health Promotion Practice. In press 